\title{
Long-Term Imaging of Cellular Forces with High Precision by Elastic Resonator Interference Stress Microscopy
}

\author{
Nils M. Kronenberg ${ }^{1}$, Philipp Liehm ${ }^{1}$, Anja Steude ${ }^{1}$, Johanna A. Knipper ${ }^{2}$, Jessica G. Borger ${ }^{2,+}$, \\ Giuliano Scarcelli ${ }^{3}$, Kristian Franze ${ }^{4}$, Simon J. Powis ${ }^{5}$, Malte C. Gather ${ }^{1, *}$ \\ 1) SUPA, School of Physics and Astronomy, University of St Andrews, St Andrews, UK \\ 2) Institute of Immunology and Infection Research, University of Edinburgh, Edinburgh, UK \\ 3) Fischell Department of Bioengineering, University of Maryland, College Park, MD, USA \\ 4) Department of Physiology, Development and Neuroscience, University of Cambridge, Cambridge, UK \\ ${ }^{5)}$ School of Medicine, University of St Andrews, St Andrews, UK
}

\begin{abstract}
Cellular forces are crucial for many biological processes but current methods to image them have limitations with respect to data analysis, resolution and throughput. Here, we present a robust approach to measure mechanical cell-substrate interactions in diverse biological systems by interferometrically detecting deformations of an elastic micro-cavity. Elastic Resonator Interference Stress Microscopy (ERISM) yields stress maps with exceptional precision and large dynamic range $(2 \mathrm{~nm}$ displacement resolution over a $>1 \mu \mathrm{m}$ range, translating into $1 \mathrm{pN}$ force sensitivity). This enables investigation of minute vertical stresses $(<1 \mathrm{~Pa})$ involved in podosome protrusion, protein specific cell-substrate interaction and amoeboid migration through spatial confinement in real time. ERISM requires no zero-force reference and avoids phototoxic effects, which facilitates force monitoring over multiple days and at high frame rates and eliminates the need to detach cells after measurements. This allows observation of slow processes like differentiation and further investigation of cells, e.g. by immunostaining.
\end{abstract}

\footnotetext{
* correspondence: mcg6@st-andrews.ac.uk

+ present address: Department of Immunology, Monash University, Melbourne, Australia
} 


\section{Introduction}

Cells sense mechanical cues and constantly probe the mechanical properties of their environment (mechano-sensing) ${ }^{1}$. Mechanical stimuli at the cellular level play an important role in cell function, e.g. migration and differentiation, as well as in tissue-level processes, e.g. morphogenesis, tumour growth and immune response ${ }^{2-7}$. Forces exerted by cells are also fundamental for many other physiological processes such as locomotion (e.g. during immune response or tumour metastasis), cell growth, wound healing, tissue formation and repair, and extracellular matrix deposition ${ }^{8-14}$.

Mechanical interaction between a cell and its substrate can occur through various mechanisms. During mesenchymal migration, lateral mechanical forces generated by the cytoskeleton are transferred to the extracellular matrix via firm focal adhesion protein complexes ${ }^{15}$. In other cases, forces act perpendicularly to the substrate and there is rapidly increasing interest in the role these vertical forces play, e.g. during the extension of special cellular processes such as podosomes ${ }^{16}$ or during amoeboid migration in spatial confinement where non-specific cell-substrate interactions such as friction and pushing are important ${ }^{17-19}$. The forces involved in these diverse mechanical interactions are expected to vary substantially in magnitude, spatial distribution and temporal evolution. Being able to monitor them in a robust and non-disruptive manner is thus critical to advancing our understanding.

Early studies obtained semi-quantitative maps of forces by direct observation of wrinkles that cell-generated forces induce in elastomer substrates ${ }^{20,21}$. Currently, the most widely used methods for imaging cellular forces (i.e. to record stress or force maps) are traction force microscopy (TFM) ${ }^{22-28}$ and the use of micro-fabricated elastic micro-pillar arrays $^{8,29,30}$. (See Ref. 31 for a review on presently available methods.) TFM and micro-pillar arrays are based on tracking displacement of fluorescent particles in hydrogels and bending of individual elastic pillars, respectively. While being powerful, these methods are indirect; in most cases the data analysis requires zero-force reference images, particularly if cell-induced substrate deformations are $<200 \mathrm{~nm}^{29,32}$. For these reference images cells have to be removed from the substrate, rendering them unavailable for further investigations. There have been attempts to obviate the need for disruptive cell removal in TFM by using highly regular meshes or grids to record cell induced substrate deformations but even under optimized conditions these approaches are limited to detecting relatively large deformations 
and require rather involved sample preparation ${ }^{32-34}$. Furthermore, fluorescence microscopy is often needed, which can be associated with photo-toxicity. Micro-pillar arrays and TFM also have limited sensitivity to vertical forces. For instance, measuring the weak vertically-directed protrusive forces exerted by podosomes has necessitated a specialized detection scheme based on atomic force microscopy $(A F M)^{16}$. What is currently missing is a more generally applicable method to image cellular forces that allows cells to be retained on the substrate for subsequent measurements and is capable of resolving weak forces with reasonable throughput.

Here, we address this need by introducing Elastic Resonator Interference Stress Microscopy (ERISM), an approach for direct, robust and non-destructive imaging of forces associated with various types of mechanical cell-substrate interactions. While most existing methods use localization microscopy or direct imaging of surface deformations, ERISM detects cell-induced substrate deformations interferometrically by using an elastic optical micro-resonator and thus provides unprecedented sensitivity. We are able to resolve not only forces exerted by cells that form firm focal adhesion contacts to the substrate but can also detect protein-specific cell-substrate interaction and quantify the much weaker vertical forces (down to piconewtons) associated with amoeboid-type cell migration through confined environments and with the protrusion of podosomes. ERISM requires no zero-force reference image, which eliminates the need to detach non-migrating cells after a measurement and enables continuous, long-term measurements of multiple cells on one substrate as well as further investigation of the cells, e.g. by immunostaining. Being a widefield imaging method, ERISM determines the local deformation at each point of the image simultaneously and requires only low light intensities, thus facilitating observation of multiple cells at once without inducing photo-damage to the cells.

\section{Results}

\section{Concept and validation of ERISM}

Cells were grown on a protein-coated elastic optical micro-cavity consisting of a layer of an ultra-soft siloxane-based elastomer (stiffness $1.3 \mathrm{kPa}$, thickness $(8 \pm 0.5) \mu \mathrm{m}$; unless stated otherwise) sandwiched between two thin and semi-transparent gold layers (Fig. 1a, b; see 
Supplementary Fig. 1a-d for details on gold-elastomer interface). Forces applied by cells were mapped with the microscope setup schematically shown in Figure 1c. Cells of interest were identified by conventional phase contrast or fluorescence microscopy (Fig. 1d). The reflection of the micro-cavity was then imaged under illumination with monochromatic light of a certain wavelength (Fig. 1e, Supplementary Fig. 1e). When a cell locally deformed the micro-cavity, a fringe pattern was formed. (At the position of each dark fringe, the incident light coupled to a resonant mode of the micro-cavity.) This fringe pattern provided a direct measure of how much the cells deformed the substrate, with each fringe corresponding to a $\approx 200 \mathrm{~nm}$ deformation. To obtain a more accurate deformation map, reflectance images were recorded for a set of different illumination wavelengths. This yielded the reflectance as a function of wavelength (Fig. 1f) and thus the local resonance wavelengths of the micro-cavity for every pixel within the field of view. The actual thickness and cell-induced deformation for each pixel were then obtained by fitting the measured resonance wavelengths with an optical model using a fast look-up table-based fitting algorithm (Fig. 1g-h).

We used AFM and a corrected Hertz model to quantify the mechanical properties of the elastic micro-cavities (Fig. 2a) ${ }^{35}$. Micro-cavities used in this study had an apparent stiffness of $3.2 \mathrm{kPa}$, in the same range as many relevant types of tissue ${ }^{36}$, and showed low stiffness variation across their surface (Fig. 2 b) and between batches (Fig. $2 \mathbf{2 c}$ ). Figure $\mathbf{2} \mathbf{d}$ shows an ERISM thickness map for a micro-cavity into which an AFM probe was indented. Using finiteelement-method (FEM) modelling we converted this indentation profile - or later, cell induced deformations - into a map of locally applied stress (Online Methods, Supplementary Fig. 1f) and found the forces obtained by the FEM model to be within $20 \%$ of the AFM set-force. By analysing the width of the AFM indentation, we estimated that the lateral resolution of ERISM is $1.6 \mu \mathrm{m}$ (Fig. 2e), which is similar or better than values achieved with super-resolution $\mathrm{TFM}^{28}$ and the latest generation arrays of sub-micrometre sized elastic pillars $^{30}$. Due to the interference-based measurement principle, ERISM measures the cell induced vertical substrate deformation with much higher resolution; in the present study, the displacement resolution was $2 \sigma_{\mathrm{rms}}=2 \mathrm{~nm}$ (limited by the surface roughness of the microcavity and pixel noise, Fig. $\mathbf{2 f}$ ). The temporal resolution, i.e. the relaxation response time of the elastic micro-cavity, was $<0.5 \mathrm{~s}$ (Fig. $\mathbf{2 g}$ ). We also tested the mechanical stability of the micro-cavity under repeated AFM indentations and under prolonged exposure to standard 
cell culture conditions (up to two weeks) and observed no significant changes (Fig. $\mathbf{2 h}$ and Supplementary Video 1).

To demonstrate the broad applicability of ERISM, we next discuss mechanical measurements for several cell types and forms of cell-substrate interaction.

\section{Localized vertical forces of podosome protrusion}

The forces macrophages apply during podosome protrusion are perpendicular to their substrate and too small to be resolved with TFM. Podosomes are small $(<1 \mu \mathrm{m})$, cylindrical, actin-based structures on the ventral side of the plasma membrane that are formed by many different cell types, e.g. invasive cancer cells and macrophages ${ }^{16}$. They play a critical role in cell migration and invasion into tissue, and it has recently been shown that they perform mechanosensing by protruding into their substrate ${ }^{16}$. Here we exploited the unprecedented vertical resolution of ERISM to resolve the protrusive forces of podosomes in primary human macrophages (Supplementary Fig. 2).

Figure 3a shows a phase contrast and an ERISM image of a macrophage on a collagencoated ERISM micro-cavity. The ERISM data showed an unstructured broad pushing of the main cell body into the substrate and pulling at the cell periphery. However, there is also a more subtle substructure underneath the cell, which we hypothesized may be associated with podosomes protruding into the substrate. To visualize this structure more clearly, we removed the broad features via spatial Fourier filtering (cut-off frequency $\approx 0.5 \mu \mathrm{m}^{-1}$; Fig. $3 a$ bottom and Fig. 3b). This revealed a large number of weak and tightly confined pushing sites (peak stress $\approx 10 \mathrm{~Pa}$; diameter, 1-2 $\mu \mathrm{m}$ ) which are surrounded by even weaker partial rings of upward pulling. Immunocytochemistry suggested that these features indeed corresponded to podosomes (Fig. 3g, see below). Although the deformations, stresses and integrated forces exerted by the podosomes were substantially smaller than what is usually observed for focal adhesions of e.g. fibroblasts 22,28 , and even though the podosome indentations were often less than $2 \mu \mathrm{m}$ apart, they were clearly resolved by ERISM. Using time-lapse measurements, we analysed the temporal evolution of the mechanical action of podosomes for a large number of podosomes and found a pronounced oscillatory behaviour (period of force oscillation $(41 \pm 10)$ s, Figure 3c-d and Supplementary Video 2), consistent with earlier studies using a specialized AFM based detection scheme ${ }^{16}$. 
We next investigated podosome behaviour during cell migration where their activity is believed to be most important ${ }^{37}$. Figure 3 e shows phase contrast and Fourier-filtered ERISM time-lapse images of the leading process of a macrophage (also see Supplementary Video 3). Over $1 \mathrm{~h}$, the leading edge expanded by $40 \mu \mathrm{m}$. This was accompanied by formation of protrusions in a region $\approx 5 \mu \mathrm{m}$ away from the leading edge of the cell. Figure $3 \mathbf{f}$ shows a kymograph of the mean applied stress along the direction of cell migration. The forces generated by podosomes in the region around the leading edge of the cell oscillated over time (Fig. 3f; red arrows indicate times of maximum pushing). The oscillations were likely associated with the speed of cell expansion as maximum podosome activity was immediately followed by stalling and in some cases even retraction (i.e. negative speed) of the leading edge (blue arrows).

Finally, to confirm that the observed structures were indeed podosomes, we fixed the cells on the micro-cavity immediately after recording an ERISM image, immunostained for actin and vinculin and performed epi-fluorescence imaging through the micro-cavity substrate (Fig. $\mathbf{3 g}$ ). This revealed a characteristic podosome dot-pattern with small actin-rich spots surrounded by vinculin rich regions (the soft substrate led to some mislocalization of vinculin $\left.{ }^{16}\right)$. The actin-rich spots co-localized with pushing sites in the stress map and the surrounding vinculin-rich regions co-localized with the weaker ring-shaped pulling.

\section{Diffuse forces during amoeboid migration in confined environments and adhesion receptor specific cell-substrate interaction}

While the podosome protrusions discussed above represent very localized cell-substrate interactions, other cell types, such as amoebae, transmit more diffuse vertical stresses through transient adhesion contacts when migrating in a three-dimensional environment ${ }^{17,38,39}$. This "amoeboid migration" is also observed in embryonic cells, cancer cells and leukocytes ${ }^{17,18,40}$. It is characterized by rapid changes in cell shape, induced by the formation of pseudopodia and plasma membrane blebs. In spatially confined environments, friction or "chimneying"-type locomotion, in which cells project themselves forward in horizontal direction by applying weak vertical forces, have been proposed as mechanisms for stress transduction ${ }^{17,18,38,41}$. However, measurements of the involved force patterns remain challenging, mainly due to the technical difficulties in detecting the underlying small vertical displacements with sufficient resolution. 
We used ERISM to study deformations induced by migrating Dictyostelium discoideum amoebae confined within a $5 \mu \mathrm{m}$-thick void as illustrated in Figure 4a. During migration, a continuous evolution of an ellipsoid push and pull pattern was observed (Fig. 4b and Supplementary Video 4). Profiles of the stresses along the longer axis of the cell are shown in Figure $\mathbf{4 c}$ to illustrate the horizontal cell migration more clearly. Importantly, horizontal migration occurs even though the detected peak stresses were $<1 \mathrm{~Pa}$.

We also applied ERISM to perform wide-field imaging of adhesion receptor specific cell-substrate interaction, specifically during the adhesion of primary murine $T$ cells. During lymphocyte migration and immunological synapse formation between T cells and antigen presenting cells, $T$ cells adhere to the extracellular matrix and to other cells ${ }^{42,43}$. The adhesion is based on a protein complex formed between Lymphocyte function-associated antigen 1 (LFA-1) - a transmembrane integrin on the surface of Tcells - and Intercellular Adhesion Molecule 1 (ICAM-1). We measured LFA-1:ICAM-1-mediated adhesion by investigating T cells on ICAM-1-coated micro-cavity substrates (Fig. 4d-e). During initial contact, a significant fraction of $T$ cells deformed the surface of these micro-cavities but no deformation was observed for T cells on uncoated and on type 1 collagen-coated microcavities (Fig. 4f), indicating that the surface interactions are protein specific. By analysing a large number of indentation events, we found that the stress sustained by the micro-cavity substrate increased with the surface concentration of ICAM-1 as more LFA-1:ICAM-1 complexes can be formed at higher ICAM-1 surface concentration (Fig. $\mathbf{4 g}$ ).

\section{Horizontal traction stresses in mesenchymal cell migration}

Next, we investigated cells that are known to apply chiefly horizontal traction forces to their substrate when in a two-dimensional environment. Although ERISM measures vertical deformations of the micro-cavity substrate, it is also highly sensitive to horizontal forces. Applying a defined horizontal force to the surface of an ERISM micro-cavity with an AFM led to local twisting of the top gold surface (Supplementary Fig. 3). The amount of twisting was directly proportional to the applied force $\left(150 \mathrm{pN}\right.$ per $\mathrm{nm}$ of twist; $R^{2}>0.99 ; n=5$ force measurements). The origin of this twisting is somewhat related to the non-linear surface wrinkling effect that early studies on cell generated traction forces have used ${ }^{20,21}$. Importantly, however, ERISM provides sufficient sensitivity to detect small and localized, 
linear surface twisting; taking into account the $2 \mathrm{~nm}$ displacement resolution of ERISM, the minimum lateral force that causes a resolvable signal above the noise floor is $300 \mathrm{pN}$.

Figure 5a shows a phase contrast image and the corresponding ERISM map of an adherent 3T3 fibroblast. The cell was fixed during the measurement and immunolabelled for actin and vinculin (Fig. $\mathbf{5 b}$ and Fig. 5c for overlay with Fourier-filtered ERISM map). At the periphery of the cell, there were multiple clearly distinguishable push/pull features (i.e. twisting; marked by pairs of blue/red arrows in the magnified inset to Fig. 5c). This twisting is consistent with earlier observations of torque being applied by focal adhesions ${ }^{26}$. Areas of high vinculin expression were located at the centre of these features, confirming that they are associated with focal adhesions transferring traction forces horizontally to the substrate. Focal adhesion complexes had sizes of $(6.1 \pm 3.4) \mu \mathrm{m}^{2}$ and transmitted horizontal forces and stresses of $(3.1 \pm 1.0) \mathrm{nN}$ and $(602 \pm 323) \mathrm{Pa}$, respectively, to the substrate ( $n=19$ focal adhesions). These values are not significantly different from reference measurements we performed using conventional TFM on stiffness-matched polyacrylamide gels (horizontal stress at focal adhesion, (386 \pm 131$) \mathrm{Pa} ; n=6$ cells; $p=0.11$, Mann-Whitney U-test). They are also consistent with earlier micro-pillar array measurements of Bovine pulmonary artery smooth muscle cells $\left(\approx 20 \mathrm{nN}\right.$ horizontal force for $4 \mu \mathrm{m}^{2}$-sized adhesion complexes on an array with fivefold larger effective stiffness $)^{29}$ and with TFM measurements of mouse embryonic fibroblasts $(\approx 500 \mathrm{~Pa}$ horizontal stress on a hydrogel substrate with a shear modulus of $\approx 2.4 \mathrm{kPa})^{26}$.

\section{Long-term imaging of mechanical activity: cell cycle, migration from tissue explants and in situ differentiation}

We then applied ERISM to monitor cell behaviour with high temporal resolution and over extended periods of time. Time-lapse data of slowly migrating 3T3 fibroblasts with a frame taken every $2 \mathrm{~s}$ revealed fast force oscillations (oscillation period, $11.3 \mathrm{~s}$; Supplementary Video 5 and Supplementary Fig. 4a-b), which is consistent with earlier observations ${ }^{5}$. Supplementary Video 6 shows a 5.5 day time-lapse video of migrating 3T3 fibroblasts ( 5 minutes per frame, over 1,600 frames total) and traces five cycles of cell division without any signs of phototoxicity or micro-cavity degradation, thus enabling the analysis of mechanical activity during and between mitotic events. During mitosis, cells rounded up which was associated with a complete loss of applied stress (Fig. 6a), consistent with earlier 
observations $^{21}$. Afterwards cells quickly reattached and began to apply forces to the substrate. Taking the total volume by which each cell indents into the substrate as a proxy for the applied force, we observed pronounced force fluctuations (period of oscillation, $(21.5 \pm 5.0) \mathrm{min}$; Fig. 6b). The amplitude of these fluctuations increased quickly after each cell division, before going through a maximum followed by a slow decay in force as the cells approached mitosis again (Fig. 6c).

Next, we applied ERISM to primary mouse fibroblasts. The robustness of the ERISM micro-cavity allowed for tissue explants to be incubated directly on the cavity for several days until cells had migrated out and onto the micro-cavity substrate (Fig. $6 \mathbf{d}$ ). We then recorded the mechanical activity of fibroblasts in close proximity to the tissue explant (Supplementary Videos 7 and 8, Supplementary Fig. 4c-e). Afterwards we stimulated the fibroblasts with TGF$\beta$ to initiate their differentiation into myofibroblasts ${ }^{10}$. Myofibroblasts express large amounts of $\alpha$-smooth muscle actin ( $\alpha$-SMA) and play a critical role in wound healing; they accumulate in the wound's granulation tissue and contribute to wound closure by applying large contractile forces ${ }^{10}$. Over the $66 \mathrm{~h}$ time course of our differentiation experiment, the mean force of cells stimulated with TGF- $\beta$ increased by about twofold, whereas no change was observed for the control group (Figure 6e; see Supplementary Fig. 4f-g for ERISM map and $\alpha$ SMA immunostaining).

\section{Discussion}

The above examples illustrate how ERISM can be used to record mechanical cell substrate interaction in a fast, robust and sensitive manner. The micro-cavity substrates used for ERISM show excellent long-term stability, which renders ERISM particularly well suited for measuring cellular forces over prolonged periods of time as demonstrated by a 5.5 day continuous timelapse consisting of over 1,600 frames. The extraction of cells from primary tissue directly on a micro-cavity facilitated investigation of primary cells without transferring them between substrates. Importantly, no zero-force reference image is required to analyse ERISM data, which eliminates the need to detach non-migrating cells after a measurement. This makes them available for subsequent studies (e.g. by immunostaining) and drastically simplifies investigation of large numbers of cells on a common substrate and at multiple time-points. To illustrate this capability, we measured forces of more than 300 cells during the 
differentiation of fibroblasts into myofibroblasts and found a twofold increase in cellular force, in line with earlier TFM measurements on a sample of much smaller size ${ }^{44}$.

Microscopy methods that require high light intensities can be problematic in terms of cellular photo-damage, in particular if repeated measurements are required during long-term investigations. The intensity needed to acquire ERISM maps is $\approx 100 \mu \mathrm{W} \mathrm{cm}-2$ much lower than the intensities required for fluorescence-based methods such as TFM. Furthermore, ERISM time-lapse data is free from drift-artefacts because the interference effect occurs within the substrate and does not require an external reference mirror.

The extreme stress sensitivity, large field of view, high dynamic range, and the timelapse capability provide unique possibilities for observing podosome protrusions during cell migration - the process during which podosome activity is believed to be most crucial. In addition, ERISM revealed that the migration of Dictyostelium discoideum under confined conditions differs fundamentally from the situation on flat substrates, for which earlier TFM

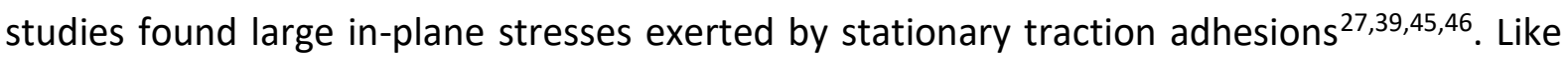
many other cell types, Dictyostelium discoideum is capable of adapting its mode of locomotion to its environment ${ }^{18,40,47-49}$. Our data suggest that in a spatially confined environment they project themselves forward in horizontal direction by applying weak vertical forces, a mechanism previously described as "chimneying"18,38,41. A recent investigation on nonadherent Walker 256 carcinosarcoma cells found that friction stresses play a pivotal role for migration through micro-channels ${ }^{17}$. While these friction stresses were too small to be resolved with TFM, they were estimated to be comparable to the stresses we measured with ERISM for Dictyostelium discoideum, indicating that ERISM may be well suited to study cell migration in micro-channels and other confined environments.

In summary, ERISM is a fast, robust and sensitive method for recording mechanical cellsubstrate interactions that is based on local interference in an elastic optical micro-cavity. It can be used to measure vertical forces $<1 \mathrm{pN}$ with a spatial resolution $<2 \mu \mathrm{m}$ and also readily detects lateral forces with $<300 \mathrm{pN}$ sensitivity. We validated ERISM by AFM, using indentation and horizontal pulling measurements, and applied it to a wide range of biological systems. ERISM can be easily combined with other microscopy modalities, such as bright-field, phase contrast or fluorescence microscopy. Its field of view can be readily adjusted by changing the microscope objective. The optics needed for ERISM can be easily integrated into a standard inverted cell culture microscope. We anticipate that the ability of ERISM to assess normal 
versus abnormal mechanical behaviour of cells quickly, together with the option to investigate multiple cells within a large field of view in real time by simply counting interference fringes, will render ERISM a very powerful tool for diagnostic applications.

\section{Acknowledgments}

The authors thank K. Venkatesan Iyer and Paul A. Reynolds for fruitful discussion, Rajesh Shahapure for TFM reference measurements, Anna L. Sobiech for illustrations and the DictyoBase for provision of AX3 strain Dictyostelium discoideum. This project has received funding from the Human Frontiers Science Program (RGY0074/2013), the Scottish Funding Council (via SUPA), the European Research Council (ERC) under the European Union's Horizon 2020 research and innovation programme (grant agreement No 640012), the EPSRC DTP (EP/L505079/1), the RS MacDonald Charitable Trust and the MRC (G1100116).

\section{Author Contributions}

N.M.K., P.L. and M.C.G. developed ERISM. N.M.K. fabricated and characterized the microcavity substrates and conducted measurements. P.L. developed the data analysis, stress map calculation and graphical data presentation. A.S. contributed to protein coating, staining and cell culture. J.A.K. designed and prepared the primary mouse fibroblast experiment. J.G.B. prepared and assisted in the T cell experiment. G.S. and K.F. performed rheometry and TFM, and contributed to AFM and general discussion. S.J.P. proposed and prepared the macrophage experiment. M.C.G. supervised the project. N.M.K. and M.C.G wrote the manuscript with input from all authors.

\section{References}

1. Geiger, B., Spatz, J. P. \& Bershadsky, A. D. Environmental sensing through focal adhesions. Nat. Rev. Mol. Cell Biol. 10, 21-33 (2009).

2. Koser, D. E. et al. Mechanosensing is critical for axon growth in the developing brain. Nat. Neurosci. 19, 1592-1598 (2016).

3. Kshitiz et al. Control of stem cell fate and function by engineering physical microenvironments. Integr. Biol. 4, 1008-1018 (2012).

4. Lo, C. M., Wang, H. B., Dembo, M. \& Wang, Y. L. Cell movement is guided by the rigidity of the substrate. Biophys. J. 79, 144-152 (2000).

5. Plotnikov, S. V., Pasapera, A. M., Sabass, B. \& Waterman, C. M. Force fluctuations 
within focal adhesions mediate ECM-rigidity sensing to guide directed cell migration. Cell 151, 1513-1527 (2012).

6. Paszek, M. J. et al. Tensional homeostasis and the malignant phenotype. Cancer Cell 8, 241-254 (2005).

7. Jannat, R. A., Robbins, G. P., Ricart, B. G., Dembo, M. \& Hammer, D. A. Neutrophil adhesion and chemotaxis depend on substrate mechanics. J. Phys. Condens. Matter 22, 194117 (2010).

8. Ricart, B. G., Yang, M. T., Hunter, C. A., Chen, C. S. \& Hammer, D. A. Measuring traction forces of motile dendritic cells on micropost arrays. Biophys. J. 101, 26202628 (2011).

9. Betz, T., Koch, D., Lu, Y.-B., Franze, K. \& Käs, J. A. Growth cones as soft and weak force generators. Proc. Natl. Acad. Sci. U. S. A. 108, 13420-13425 (2011).

10. Tomasek, J. J., Gabbiani, G., Hinz, B., Chaponnier, C. \& Brown, R. A. Myofibroblasts and mechano-regulation of connective tissue remodelling. Nat. Rev. Mol. Cell Biol. 3, 349-363 (2002).

11. Mertz, A. F. et al. Scaling of traction forces with the size of cohesive cell colonies. Phys. Rev. Lett. 108, 198101 (2012).

12. Lemmon, C. A., Chen, C. S. \& Romer, L. H. Cell traction forces direct fibronectin matrix assembly. Biophys. J. 96, 729-738 (2009).

13. Brugués, A. et al. Forces driving epithelial wound healing. Nat. Phys. 10, 683-690 (2014).

14. Tambe, D. T. et al. Collective cell guidance by cooperative intercellular forces. Nat. Mater. 10, 469-475 (2011).

15. Case, L. B. \& Waterman, C. M. Integration of actin dynamics and cell adhesion by a three-dimensional, mechanosensitive molecular clutch. Nat. Cell Biol. 17, 955-963 (2015).

16. Labernadie, A. et al. Protrusion force microscopy reveals oscillatory force generation and mechanosensing activity of human macrophage podosomes. Nat. Commun. 5, 5343 (2014).

17. Bergert, M. et al. Force transmission during adhesion-independent migration. Nat. Cell Biol. 17, 524-529 (2015).

18. Liu, Y. J. et al. Confinement and low adhesion induce fast amoeboid migration of slow mesenchymal cells. Cell 160, 659-672 (2015).

19. Toyjanova, J., Flores-Cortez, E., Reichner, J. S. \& Franck, C. Matrix confinement plays a pivotal role in regulating neutrophil-generated tractions, speed, and integrin utilization. J. Biol. Chem. 290, 3752-3763 (2015).

20. Harris, A. K., Wild, P. \& Stopak, D. Silicone rubber substrata: A new wrinkle in the study of cell locomotion. Science 208, 177-179 (1980).

21. Burton, K. \& Taylor, D. L. Traction forces of cytokinesis measured with optically modified elastic substrata. Nature 385, 450-454 (1997).

22. Dembo, M. \& Wang, Y. L. Stresses at the cell-to-substrate interface during locomotion of fibroblasts. Biophys. J. 76, 2307-2316 (1999).

23. Schwarz, U. S. et al. Calculation of forces at focal adhesions from elastic substrate data: the effect of localized force and the need for regularization. Biophys. J. 83, 1380-1394 (2002).

24. Sabass, B., Gardel, M. L., Waterman, C. M. \& Schwarz, U. S. High resolution traction force microscopy based on experimental and computational advances. Biophys. J. 94, 
207-220 (2008).

25. Hur, S. S., Zhao, Y., Li, Y. S., Botvinick, E. \& Chien, S. Live cells exert 3-dimensional traction forces on their substrata. Cell. Mol. Bioeng. 2, 425-436 (2009).

26. Legant, W. R. et al. Multidimensional traction force microscopy reveals out-of-plane rotational moments about focal adhesions. Proc. Natl. Acad. Sci. U. S. A. 110, 881-886 (2013).

27. Álvarez-González, B. et al. Three-dimensional balance of cortical tension and axial contractility enables fast amoeboid migration. Biophys. J. 108, 821-832 (2015).

28. Colin-York, H. et al. Super-resolved traction force microscopy (STFM). Nano Lett. 16, 2633-2638 (2016).

29. Tan, J. L. et al. Cells lying on a bed of microneedles: an approach to isolate mechanical force. Proc. Natl. Acad. Sci. U. S. A. 100, 1484-1489 (2003).

30. Wolfenson, H. et al. Tropomyosin controls sarcomere-like contractions for rigidity sensing and suppressing growth on soft matrices. Nat. Cell Biol. Biol. 18, 33-42 (2015).

31. Polacheck, W. J. \& Chen, C. S. Measuring cell-generated forces: a guide to the available tools. Nat. Methods 13, 415-423 (2016).

32. Polio, S. R., Rothenberg, K. E., Stamenovic, D. \& Smith, M. L. A micropatterning and image processing approach to simplify measurement of cellular traction forces. Acta Biomater. 8, 82-88 (2012).

33. Bergert, M. et al. Confocal reference free traction force microscopy. Nat. Commun. 7, 12814 (2016).

34. Balaban, N. Q. et al. Force and focal adhesion assembly: a close relationship studied using elastic micropatterned substrates. Nat. Cell Biol. 3, 466-472 (2001).

35. Dimitriadis, E. K., Horkay, F., Maresca, J., Kachar, B. \& Chadwick, R. S. Determination of elastic moduli of thin layers of soft material using the atomic force microscope.

Biophys. J. 82, 2798-810 (2002).

36. Levental, I., Georges, P. C. \& Janmey, P. A. Soft biological materials and their impact on cell function. Soft Matter 2, 1-9 (2007).

37. Calle, Y., Burns, S., Thrasher, A. J. \& Jones, G. E. The leukocyte podosome. Eur. J. Cell Biol. 85, 151-157 (2006).

38. Charras, G. \& Paluch, E. Blebs lead the way: how to migrate without lamellipodia. Nat. Rev. Mol. Cell Biol. 9, 730-736 (2008).

39. Bastounis, E. et al. Both contractile axial and lateral traction force dynamics drive amoeboid cell motility. J. Cell Biol. 204, 1045-1061 (2014).

40. Lämmermann, T. et al. Rapid leukocyte migration by integrinindependent flowing and squeezing. Nature 453, 51-55 (2008).

41. Malawista, S. E. \& de Boisfleury Chevance, A. Random locomotion and chemotaxis of human blood polymorphonuclear leukocytes (PMN) in the presence of EDTA: PMN in close quarters require neither leukocyte integrins nor external divalent cations. Proc. Natl. Acad. Sci. U. S. A. 94, 11577-11582 (1997).

42. Friedl, P. \& Weigelin, B. Interstitial leukocyte migration and immune function. Nat. Immunol. 9, 960-969 (2008).

43. Kinashi, T. Intracellular signalling controlling integrin activation in lymphocytes. Nat. Rev. Immunol. 5, 546-559 (2005).

44. Chen, J., Li, H., SundarRaj, N. \& Wang, J. H.-C. Alpha-smooth muscle actin expression enhances cell traction force. Cell Motil. Cytoskeleton 64, 248-57 (2007). 
45. Delanoe-Ayari, H., Rieu, J. P. \& Sano, M. 4D traction force microscopy reveals asymmetric cortical forces in migrating dictyostelium cells. Phys. Rev. Lett. 105, 248103 (2010).

46. Alamo, J. C. del et al. Three-dimensional quantification of cellular traction forces and mechanosensing of thin substrata by fourier traction force microscopy. PLoS One 8, e69850 (2013).

47. Renkawitz, J. et al. Adaptive force transmission in amoeboid cell migration. Nat. Cell Biol. 11, 1438-1443 (2009).

48. Petrie, R. J., Gavara, N., Chadwick, R. S. \& Yamada, K. M. Nonpolarized signaling reveals two distinct modes of 3D cell migration. J. Cell Biol. 197, 439-455 (2012).

49. Yoshida, K. \& Soldati, T. Dissection of amoeboid movement into two mechanically distinct modes. J. Cell Sci. 119, 3833-3844 (2006). 
a

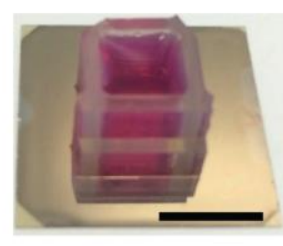

C

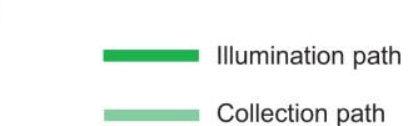

Tuneable

light source

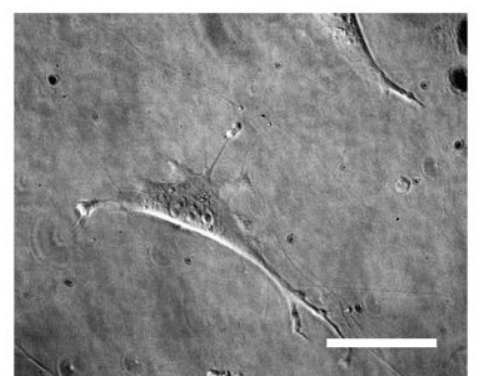

b

$$
\begin{array}{r}
15 \mathrm{~nm} \text { Au with } \\
\text { bio coating } \\
\text { Elastomer } \\
10 \mathrm{~nm} \text { Au on } \\
\text { glass substrate }
\end{array}
$$

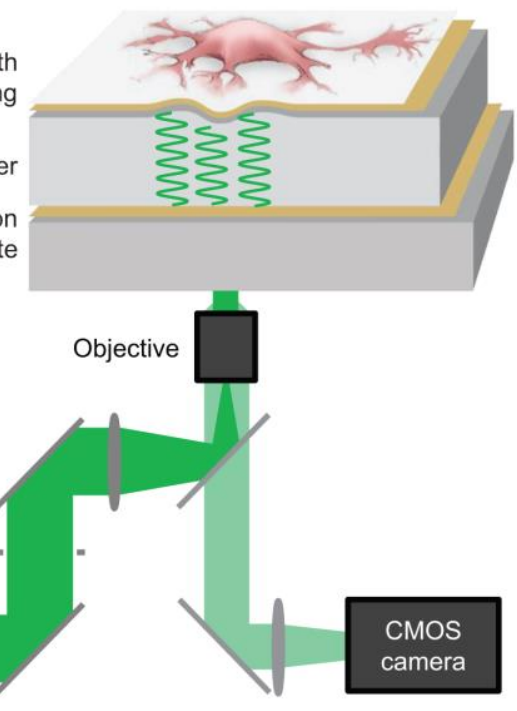

e

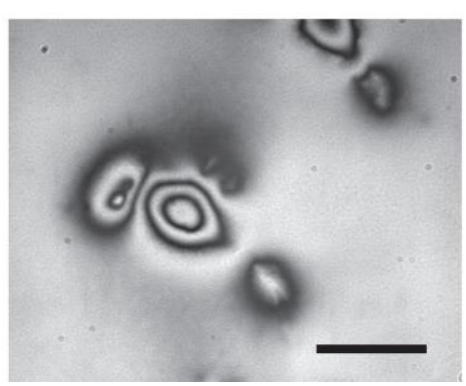

f

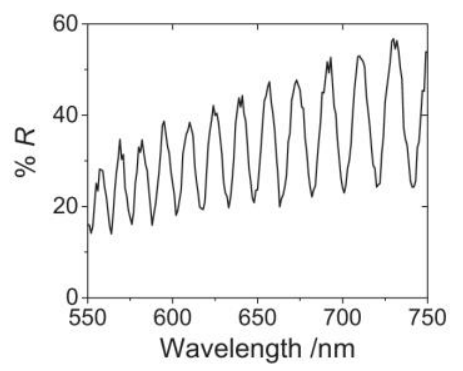

g

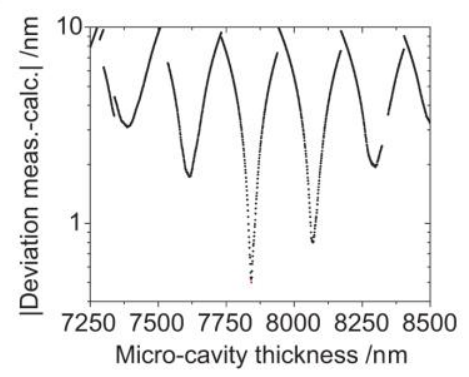

h

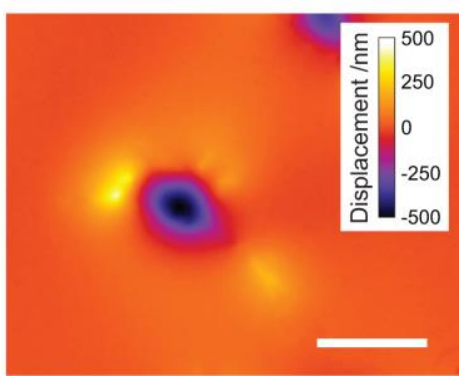

Figure 1 | Elastic Resonator Interference Stress Microscopy (ERISM). (a) Photograph of the micro-cavity with a silicone chamber filled with cell medium mounted on top. (b) Sketch of layered structure of the micro-cavity with elastomer layer sandwiched between two ultra-thin gold mirrors. (c) Schematic of the wide-field optical readout system with tuneable light source providing monochromatic illumination and epi-collection to record spatially resolved images of micro-cavity reflectance. (d) Phase contrast image of a cell attached to the microcavity surface. (e) Co-registered monochromatic reflectance image of micro-cavity at $655 \mathrm{~nm}$ wavelength of illumination. (f) Wavelength dependence of the reflectance for one representative pixel within the field of view. (g) Absolute deviation between the measured resonance wavelengths of a representative pixel and the resonance positions calculated for different micro-cavity thicknesses. The minimum deviation corresponds to the actual thickness, in this example $7843 \mathrm{~nm}$. Inset: Detail of area highlighted in main panel. (h) ERISM map obtained from the reflectance data, showing deformation induced by cell from (d). Outline of cell in black. Scale bars, (a) $1 \mathrm{~cm},(d, e, h) 50 \mu \mathrm{m}$. 


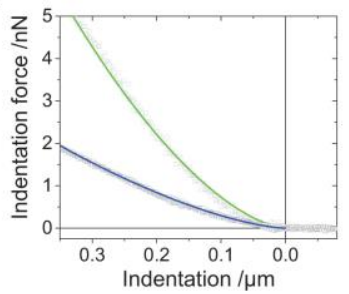

e

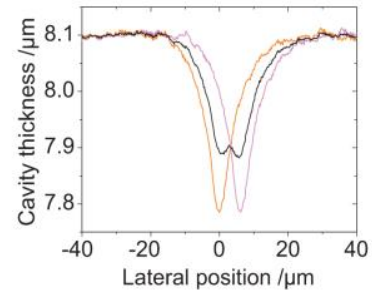

b

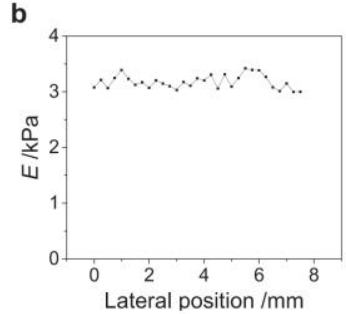

f

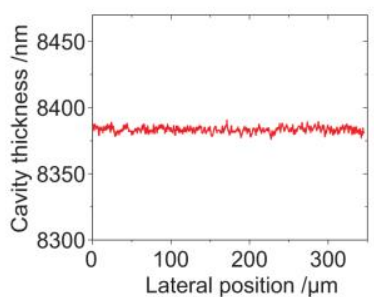

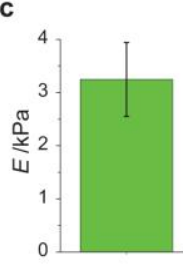

g

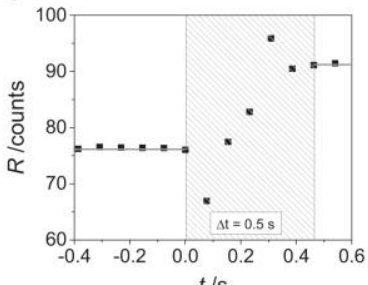

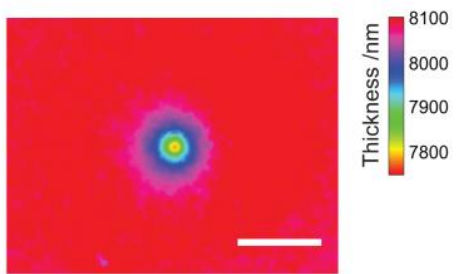

h

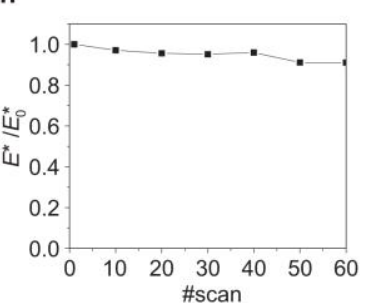

Figure 2 | Validation of ERISM by atomic force microscopy (AFM). (a) Force-distance curves (open symbols) obtained by AFM micro-indentation with a $17 \mu \mathrm{m}$-diameter glass sphere and corrected Hertz model fits to the data for pristine elastomer (blue line) and for complete micro-cavity (green line). (b) Apparent stiffness obtained from Hertz model fit along the surface of a micro-cavity; $\mathrm{n}=31$ measurements, standard deviation, $0.12 \mathrm{kPa}$. (c) Mean stiffness and standard deviation for micro-cavities produced in different batches ( $n=5$ independent measurements). (d) ERISM thickness map of micro-cavity upon AFM micro-indentation with a $17 \mu \mathrm{m}$-diameter glass sphere (set-force, $5 \mathrm{nN}$ ). (e) Vertical profile through thickness map (orange), same profile shifted horizontally by $6.2 \mu \mathrm{m}$ (magenta) and superposition of both profiles (black), the latter still clearly showing two separate minima. Subtracting the contact diameter of the sphere $(4.6 \mu \mathrm{m})$ yields a Sparrow resolution limit for the lateral resolution of ERISM of $1.6 \mu \mathrm{m}$. (f) Representative vertical profile along an ERISM thickness map of a pristine, non-indented microcavity. (g) Reflectance change of elastic micro-cavity when quickly $(10 \mathrm{~ms})$ removing the AFM indenter from a $600 \mathrm{~nm}$ indentation. (h) Relative change in the apparent stiffness of the cavity over multiple AFM indentations with a $10 \mu \mathrm{m}$-diameter sphere and an indentation depth of $600 \mathrm{~nm}$. Scale bar (d) $25 \mu \mathrm{m}$. 
a
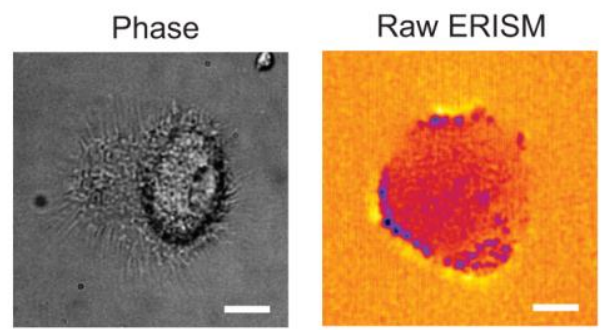

Fourier filtered ERISM

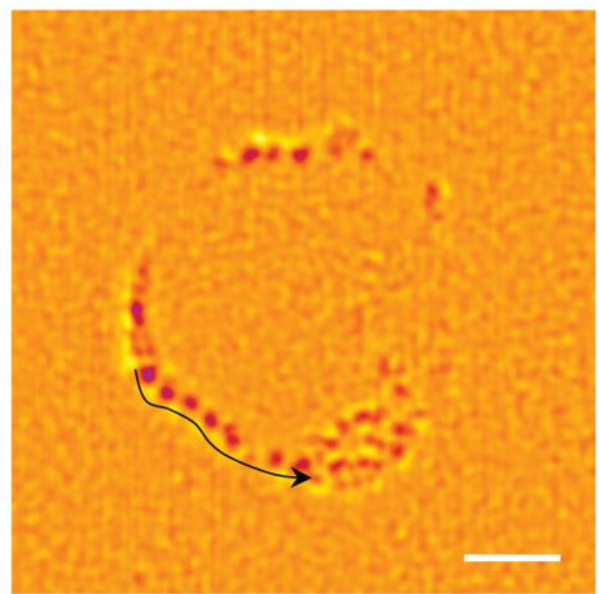

e
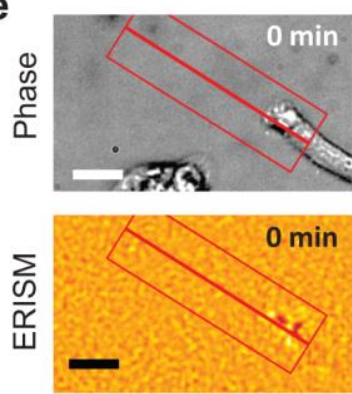

f
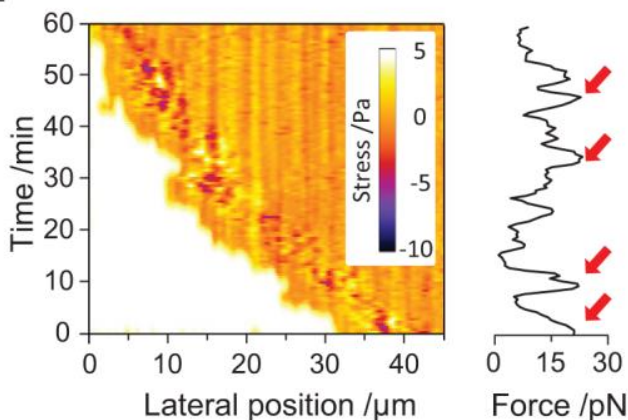
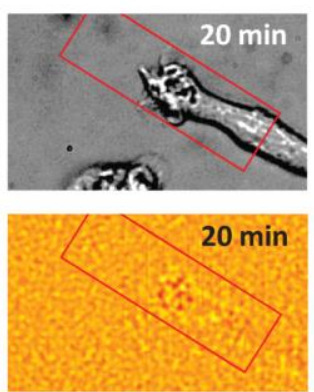

$20 \mathrm{~min}$
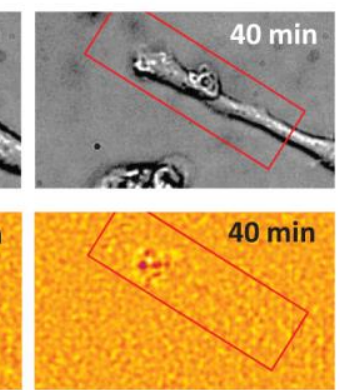

b

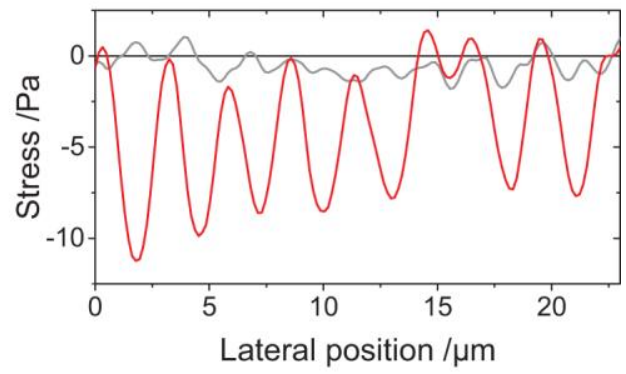

C

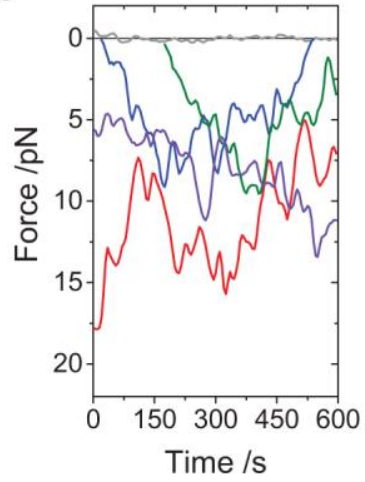

d

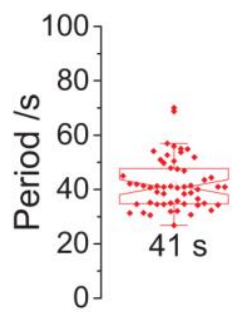

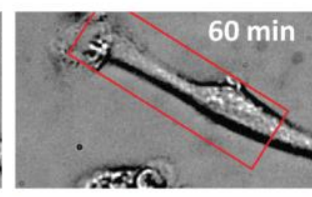

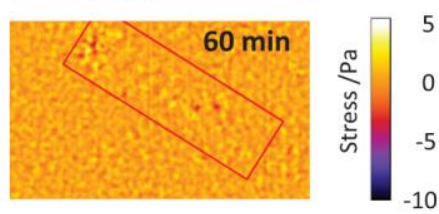

g
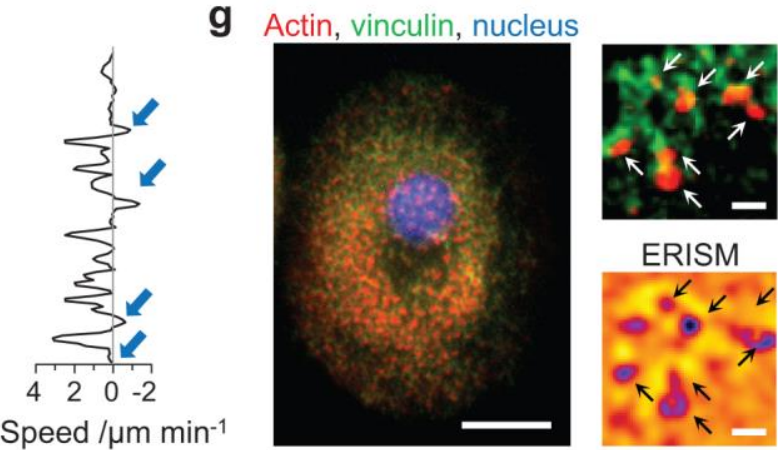

Figure 3 | Investigation of podosome protrusions by ERISM. (a) Phase contrast (upper left), ERISM (upper right) and spatial Fourier-filtered ERISM image (bottom) of a macrophage. (b) Stress profile along the long black arrow in the Fourier-filtered ERISM map in (a) (red line) and the typical noise in a region outside the cell area (grey line). (c) Temporal evolution of the force applied by several podosome protrusions and for a control area away from a podosome (grey line). Data extracted from a $1 \mathrm{~h}$ ERISM time-lapse measurement with a frame every $5 \mathrm{~s}$, calculating the force by integrating the applied stress over the area of the respective podosome. (d) Tukey boxplot showing oscillation periods for $n=50$ podosomes from five different cells. Each data point represents 
one podosome. (e) Investigation of podosome protrusion during cell expansion. Top row: Phase contrast images showing an elongating macrophage for four different time points. Bottom row: Corresponding Fourier filtered ERISM maps. (f) Left: Kymograph analysis of applied stress along bold red line in (e) averaged over a $7 \mu \mathrm{m}$-wide area above and below the line as illustrated by red rectangles in (e). White areas in kymograph are areas into which the cell has not yet expanded. Centre: Temporal evolution of the total force applied by all podosomes in leading edge of macrophage. Right: Temporal evolution of expansion speed of the cell. (g) Epi-fluorescence image of fixed macrophage with staining for actin, vinculin and nuclear DNA. Insets, magnified epi-fluorescence (top) and co-located ERISM map (bottom) with arrows indicating positions of individual podosomes. Scale bars in insets of (g) $2 \mu \mathrm{m}$, all other scale bars $10 \mu \mathrm{m}$. Images are representative of three independent experiments. 
a

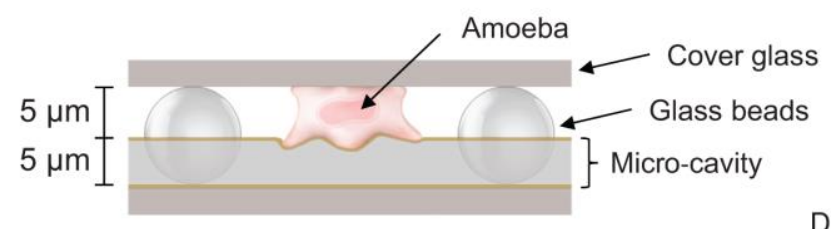

b
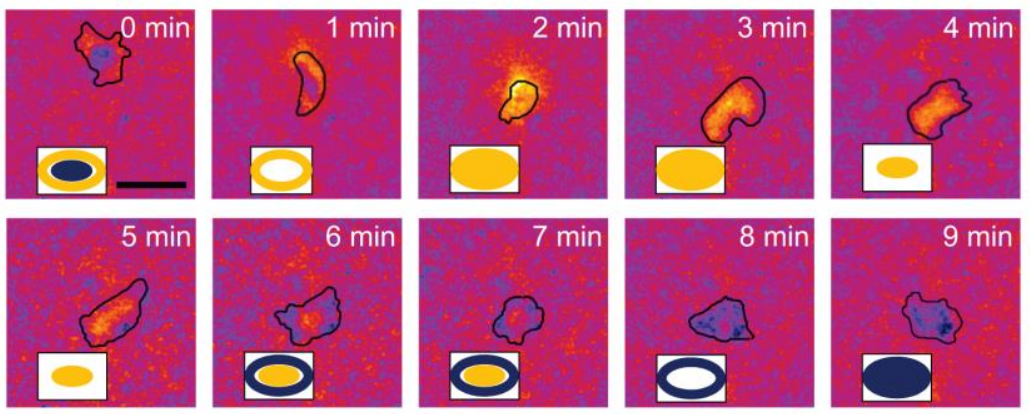

d

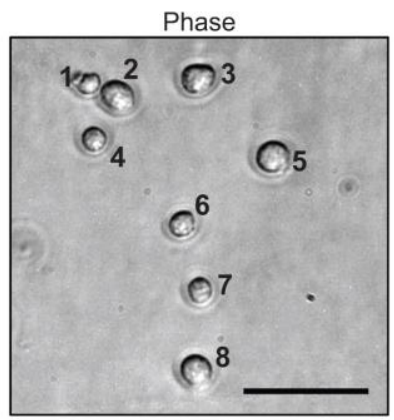

ERISM

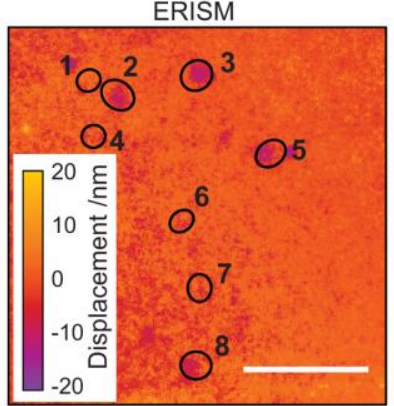

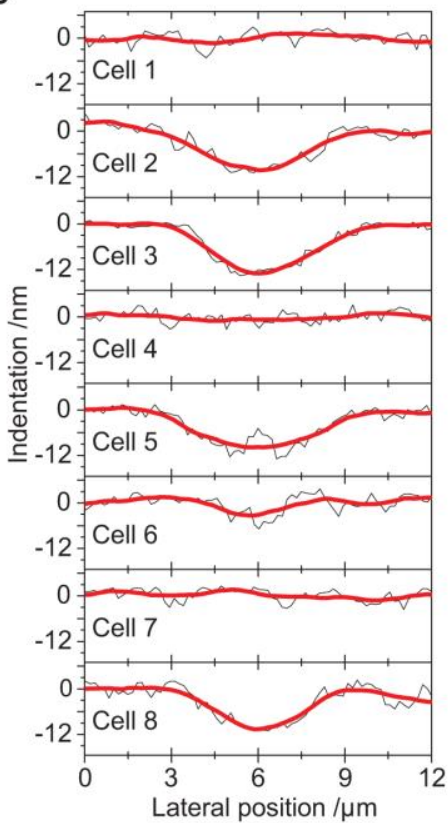

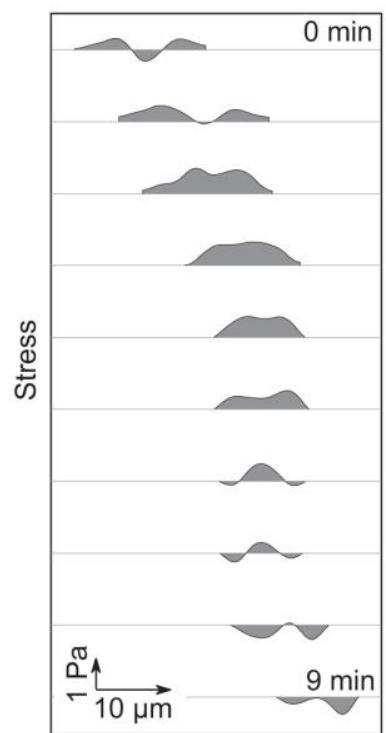

Lateral position

f

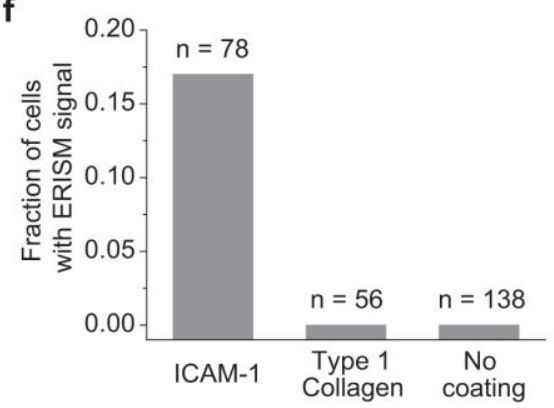

g

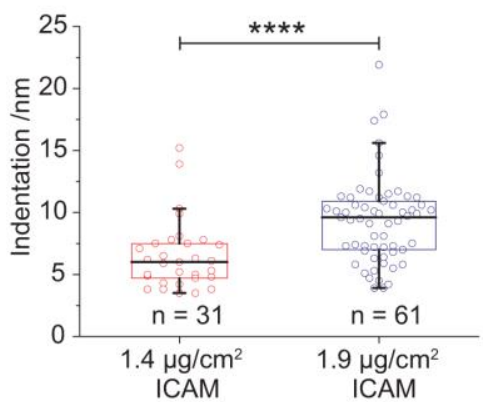

Figure 4 | ERISM for investigation of amoeboid migration in confined space and protein-specific cell-substrate interaction. (a) Illustration of $5 \mu \mathrm{m}$ void formed on top of a $5 \mu \mathrm{m}$ thick micro-cavity substrate by using $10 \mu \mathrm{m}$ diameter glass beads and a glass coverslip. (b) Temporal evolution of substrate deformation generated by a Dictyostelium discoideum amoeba migrating through the void. The boundary of the cell is indicated in black. The insets show schematic illustrations of the displacement pattern at each time point. (c) Profiles of stress along the long axis of the cell (with $8 \mu \mathrm{m}$ bandwidth spatial averaging to filter surface roughness). (d) Phase contrast image and ERISM map of LFA-1 integrin presenting activated primary murine T cells on a micro-cavity substrate coated with ICAM-1. (e) Indentation profiles for the T cells shown in (d) as measured (black) and spatially averaged $(5 \mu \mathrm{m}$ bandwidth, red). (f) Fraction of Tcells causing an ERISM signal for ICAM-1-coated, type 1 collagen-coated and uncoated substrates. (g) Tukey boxplot of indentation upon contact with activated 
T cells for micro-cavity substrates coated with different ICAM-1 surface concentrations. As data in groups was not normally distributed, groups were compared using the Mann-Whitney U-test (**** $<0.0001)$. Scale bar in (b) $15 \mu \mathrm{m}$, other scale bars $25 \mu \mathrm{m}$. $\mathrm{n}$, number of indentations analysed. Images and data are representative of three independent observations. 
a

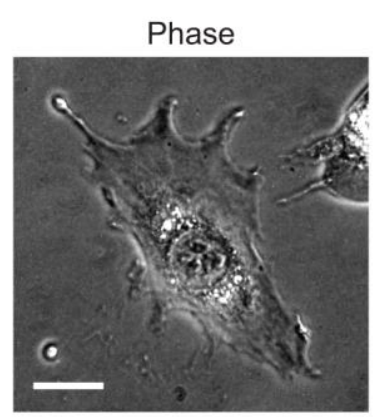

ERISM

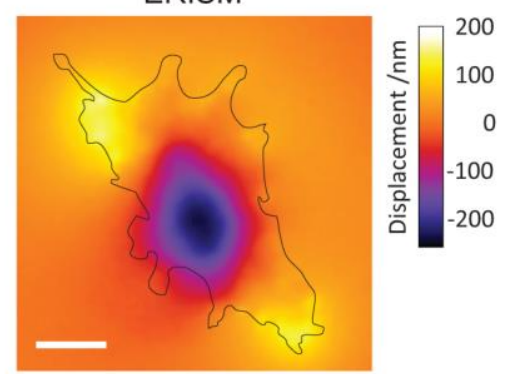

b

Actin, vinculin, nucleus

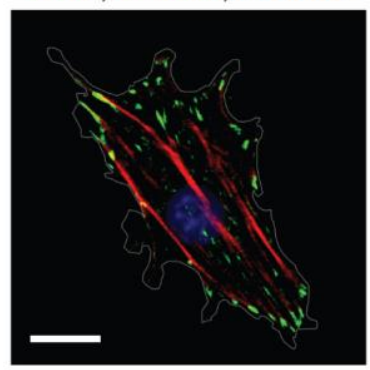

C

Fourier filtered ERISM, vinculin

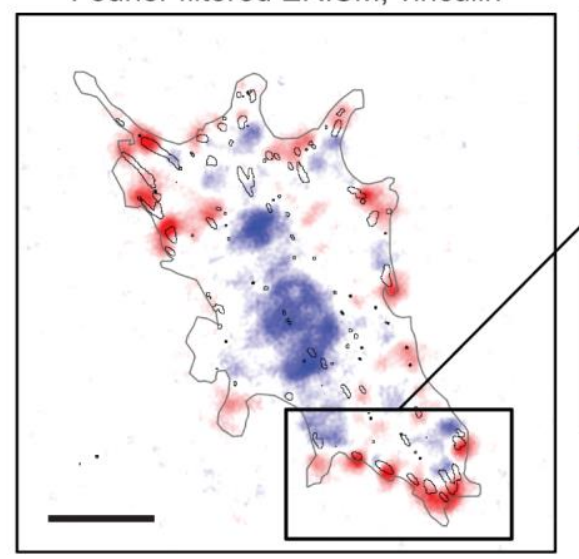

Fourier filtered ERISM, vinculin (magnified)

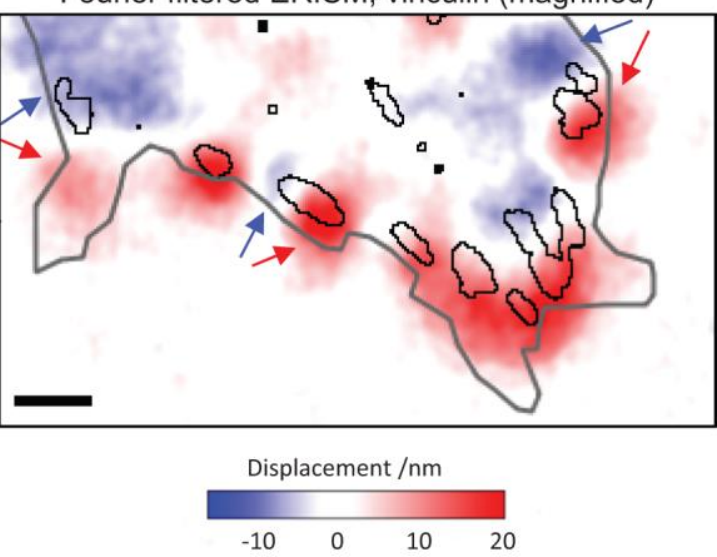

Figure 5 | Analysis of the horizontal stress applied by adherent 3T3 fibroblasts using ERISM. (a) Phase contrast image and false colour map of the micro-cavity deformation generated by a 3T3. The cell boundary is shown in grey. (b) Epi-fluorescence image of same cell following fixation and staining for actin, vinculin and nuclear DNA. (c) Fourier-filtered ERISM data from (a). Cell outline in grey. Black lines indicate vinculin-rich areas, overlay of data from (b). All scale bars $25 \mu \mathrm{m}$; except for inset to (c) $5 \mu \mathrm{m}$. Images are representative of five independent experiments. 
a

$-1 \mathrm{~h}$
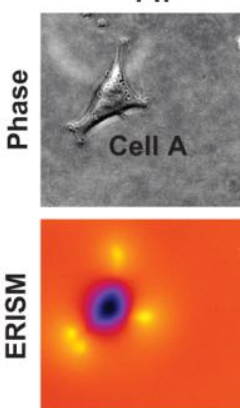

b

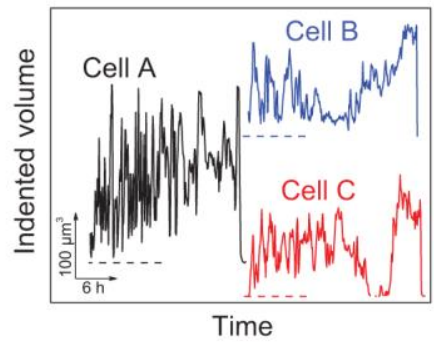

$1 \mathrm{~h}$
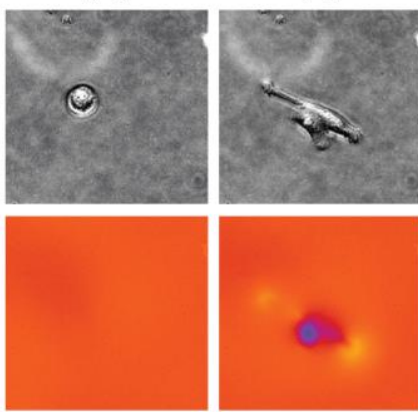

C

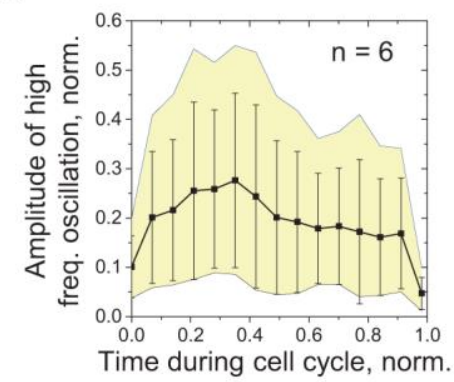

$2 \mathrm{~h}$
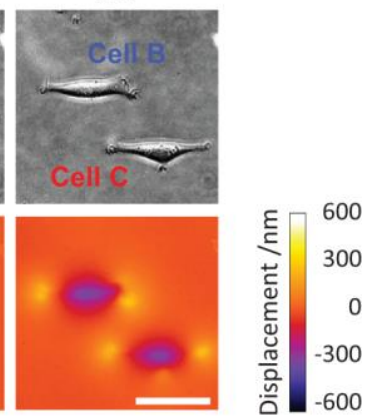

$-600$ d

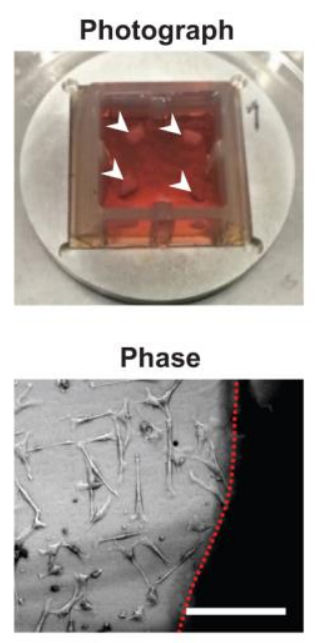

e

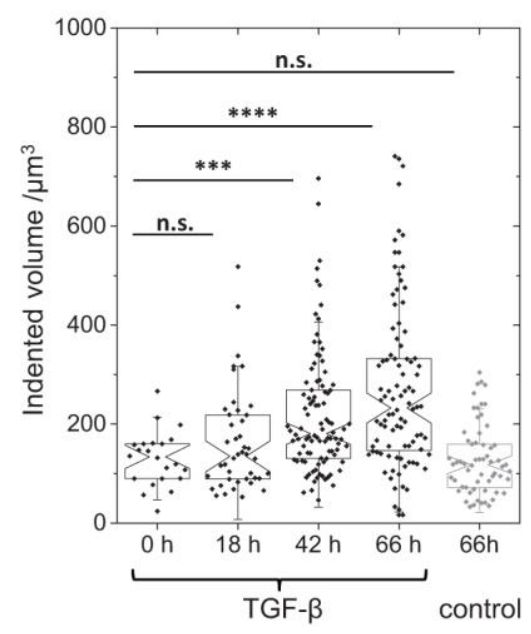

Figure 6 | ERISM for long-term time lapse studies of the mechanical activity of cells. (a) Representative phase contrast and ERISM images from a 1,600 frame time-lapse series which recorded the mechanical activity of 3T3 fibroblasts over 5.5 days. Shown is one of several cell-division events with mother cell (Cell A) and two daughter cells (Cell B and C). Images are representative of seven independent observations. (b) Temporal evolution of the mechanical activity of Cells A-C over the course of their cell cycles. Traces show a high-frequency oscillatory behaviour that changes in amplitude over time. (Traces vertically displaced for clarity; dashed lines indicate zero levels for each trace.) (c) Analysis of the oscillatory mechanical activity of $n=6$ fibroblasts during their cell cycle. Shown are the mean (black squares), standard deviation (whiskers) and minimum and maximum amplitude (yellow area) of the oscillation amplitude. (d) Photograph of four pieces of mouse skin (marked by arrow heads) incubated directly on a micro-cavity substrate and bright-field microscopy image of primary fibroblasts migrating out of the skin (shadowed area separated by red dashed line) on day four of incubation. (e) Investigation of TGF- 
$\beta$-induced differentiation of primary mouse fibroblasts into myofibroblasts. Tukey boxplot of mechanical activity before ( $0 h, n=22$ cells) and during ( $18 h, n=42$ cells; $42 h, n=99$ cells; $66 h, n=92$ cells) incubation with $10 \mathrm{ng}$ $\mathrm{mL}^{-1}$ TGF- $\beta$ and with media alone (control group, $\mathrm{n}=59$ cells). Each dot represents one cell. As data in groups was not normally distributed, groups were compared using the Mann-Whitney U-test $\left({ }^{* * *} p<0.001\right.$; **** $p<0.0001$ ). Scale bars, $50 \mu \mathrm{m}$ in (a) and $200 \mu \mathrm{m}$ in (d). 